\title{
A brief introduction to the ABINIT software package
}

\author{
Xavier Gonze*,I, Gian-Marco Rignanese ${ }^{\mathrm{I}}$, Matthieu Verstraete ${ }^{\mathrm{I}}$, Jean-Michel Beuken ${ }^{\mathrm{I}}$, Yann Pouillon ${ }^{\mathrm{I}}$, Razvan Caracas ${ }^{\mathrm{II}}$, \\ Francois Jollet ${ }^{\mathrm{III}}$, Marc Torrent ${ }^{\mathrm{III}}$, Gilles Zerah ${ }^{\mathrm{III}}$, Masayoshi Mikami ${ }^{\mathrm{IV}}$, Philippe Ghosez ${ }^{\mathrm{V}}$, Marek Veithen ${ }^{\mathrm{V}}$, \\ Jean-Yves Raty ${ }^{\mathrm{V}}$, Valerio Olevano ${ }^{\mathrm{VI}}$, Fabien Bruneval ${ }^{\mathrm{VI}}$, Lucia Reining ${ }^{\mathrm{VI}}$, Rex Godby ${ }^{\mathrm{VII}}$, Giovanni Onida ${ }^{\mathrm{VIII}}$, \\ Donald R. Hamann ${ }^{\mathrm{IX}}$ and Douglas C. Allan ${ }^{\mathrm{X}}$ \\ I Université Catholique de Louvain, place Croix du sud 1, 1348 Louvain-la-neuve, Belgium \\ II University of Minnesota, Washington, Ave. SE, 421, Minneapolis MN 55455, USA \\ III Département de Physique Théorique et Appliquée, CEA-DAM-Ile de France, BP 12, 91680 Bruyères le Châtel, France \\ IV Mitsubishi Chemical Group Science and Technology Research Center, Inc., 1000 Kamoshida-cho, Aoba-ku, Yokohama, 227-8502, Japan \\ $\mathrm{v}$ Université de Liège, Allée du 6 août 17, 4000 Liège, Belgium \\ VI Laboratoire des Solides Irradiés UMR 7642, CNRS - CEA École Polytechnique, 91128 Palaiseau, France \\ VII Department of Physics, University of York, York YO10 5DD, United Kingdom \\ VIII INFM and Dipartimento di Fisica dell'Universita di Milano, via Celoria 16, 20133 Milano, Italy \\ IX Bell Laboratories, Lucent Technologies, Mountain Ave. 600, 07974, USA \\ X Corning Incorporated, SP-FR-05, Sullivan Park, Corning, NY 14831, USA
}

Received June 23, 2004; accepted August 17, 2004

Computational crystallography / Vibrational properties / Elastic properties / Specific heat/ABINIT Computer program

\begin{abstract}
A brief introduction to the ABINIT software package is given. Available under a free software license, it allows to compute directly a large set of properties useful for solid state studies, including structural and elastic properties, prediction of phase (meta)stability or instability, specific heat and free energy, spectroscopic and vibrational properties. These are described, and corresponding applications are presented. The emphasis is also laid on its ease of use and extensive documentation, allowing newcomers to quickly step in.
\end{abstract}

\section{Introduction}

The ABINIT software project started in 1997. The initial goal was the delivery, under a Free Software license, of a software package for the $a b$ initio study of material properties. Such a license had been crucial to the success of the Linux Operating System, and we felt that the community of material scientists would appreciate a similar project.

The first publicly available version of ABINIT was released in December 2000. Since then, the developers community has grown from a dozen early enthusiasts, to about fifty people. The user community, about ten times larger, has grown at about the same rate.

Because of its large developer group, the computational capabilities of ABINIT cover a wide spectrum of properties: computation of equilibrium cell parameters and atomic positions, vibrational properties (for infra-red and Raman analysis), prediction of phase (meta)stability or

* Correspondence author (e-mail: gonze@pcpm.ucl.ac.be) instability, elastic properties, dielectric and piezoelectric properties, non-linear optical properties, thermodynamic behaviour (entropy, free energy, specific heat), electronic properties (metal/insulator characterization), magnetic properties, space group analysis ...

However, ABINIT is also especially interesting because of its ease of use and its accompanying high-level documentation: the user's point of view has been at the center of the project since the beginning. Indeed, developers are also users or lecturers, and many of them have taken time to improve ABINIT in order to make their own life easier.

In the next section, the theoretical framework will be briefly presented, the capabilities of ABINIT will be illustrated, and the properties accessible directly by ABINIT will be described. The user's point of view will be developed in the third section, where the availability of ABINIT will also be described. Due to space limitations and the focused interest of this presentation, we will not attempt to describe the software engineering techniques used in ABINIT, or to analyse ABINIT parallelism and performances. Also, some capabilities of ABINIT, less relevant for crystallographic and mineralogical studies, have been omitted. To complete the present paper, the interested reader might consult an earlier description of the project [1], visit the Web site [2], or examine recent selected papers [3-10].

\section{Properties and applications}

\subsection{The underlying theoretical framework}

ABINIT's main program is mostly based on Density Functional Theory (DFT) [11, 12]. For response functions, like vibrations, dielectric or piezoelectric properties, the specialized version of DFT, the Density Functional Perturbation 
Theory [13-17] has been implemented, providing efficiency and ease of use. See the special review article on lattice dynamics of crystals and related properties, in the present issue [18]. These two aspects of DFT provide the core of ABINIT's capabilities. However, for selected electronic properties, in particular the prediction of electronic band gaps (and the metallic or insulating character), Density Functional Theory is notoriously unreliable. In ABINIT, such electronic properties can be computed accurately thanks to an implementation of the many-body perturbation theory, the so-called "GW approximation" [19]. We will now focus on DFT, leaving the description of GW to subsection 2.3.

Density Functional Theory, which is in principle exact, must be approximated to give a tractable scheme. Different approximations to the exchange-correlation energy functional have been implemented, including different flavors of the Local Density Approximation and Generalized Gradient Approximation [1]. With such approximations, bond lengths and angles are generally predicted within a few percent.

ABINIT is based on a plane-wave expansion of the electronic wavefunctions, with a periodic representation of the system in a box under periodic boundary conditions. This representation is especially suitable for crystal studies: the box is taken as the primitive unit cell. Taking a non-primitive cell (or super-cell) allows the study of systems in which the translational symmetry is reduced. Thus, one can address trace elements, crystal defects, interfaces and, as soon as images of the system in the neighboring supercell are sufficiently far from each other, surfaces, or systems placed in vacuum. To give some idea of the size of allowed systems, let us mention that ABINIT has been used for testing purposes up to 250 atoms, while most routine ABINIT DFT studies do not exceed 50 atoms, and even, in the case of the more demanding GW studies, not even a dozen atoms.

Pseudopotentials allow one to remove the explicit treatment of core electrons, and to focus on the formation of the bonds and response properties of the valence electrons. ABINIT has an extensive library of norm-conserving pseudopotentials, for the whole periodic table. Recently, the projector-augmented wave technique [20] has been implemented.

Metals as well as insulators can be readily studied: grids of wavevectors needed to sample the electronic contributions to the density in the Brillouin zone are automatically generated. For metals, different smearing schemes allow to keep the number of such wavevectors reasonably small. Magnetism is properly dealt with: ferromagnetic as well as anti-ferromagnetic materials are as accessible as non-magnetic ones. Calculations including spin-orbit coupling and non-collinear magnetism are also possible.

\subsection{Atomic geometry}

For a given geometry, ABINIT is able to compute analytically the forces on atoms and stresses on the cell. Such information allows one to start the optimization of atomic positions and unit cell parameters: new trial geometries are generated, for which the corresponding forces and stresses are evaluated. Based on the repetition of this basic step, different algorithms are available to optimize the geometry [1]. After a few dozen iterations, in general, the requirements on the residual forces and stresses, as defined by the users, are met, and the geometry is considered converged. As mentioned previously, the typical accuracy on such geometry parameters is on the order of a few percent.

In some cases, the user is interested in the classical dynamics of its system: ABINIT can rely on a Nosé thermostat to sample the canonical ensemble and simulate the behavior of the system at different temperatures (liquid state, generation of amorphous state ...). Sometimes, the user will want to explore the extremely large space of possible atomic configurations (including large atomic rearrangements). The Langevin dynamics provides an interesting methodology for such studies [1].

In the treatment of the crystalline state, symmetries play an important role. In ABINIT, a database of the 230 (Fedorov) space groups and the 1191 (Shubnikov) magnetic groups has been set up. Thanks to this database, different operations can be performed: reconstruction of the atomic coordinates in the full primitive cell from its irreducible part, identification of the space group if all atomic coordinates are given, automatic determination of the symmetry operations. This database follows the conventions used in the International Tables for Crystallography.

\subsection{Electronic properties}

Electronic charge densities are one basic output of DFT. In ABINIT, because of the use of pseudopotentials, one focuses on valence charge densities, the core electrons being assumed frozen. In the interstitial or bonding regions, such a charge density is quite accurate, while close to the nucleus, the modifications due to pseudopotentials should not be forgotten.

As already mentioned, the use of DFT to predict the band structure is questionable, and should therefore be restricted to qualitative analyses. This is particularly critical for the investigation of the metallic/insulator behavior, or the quantitative prediction of the band gap (the so-called "band gap problem" of DFT).

The GW methodology, on the contrary, is generally able to achieve a very satisfactory description of the band structure and their band gaps, with typical discrepancy less than $0.2 \mathrm{eV}$ with respect to the experimental data. A GW calculation has to be performed for a fixed geometry (preferably the optimized one, or the experimental one), after a DFT calculation has already been performed (DFT electronic wavefunctions are needed), in a separate module of ABINIT.

Other electronic properties are available: electronic Density-Of-States, its projection on atomic states, Bader charges, Hirshfeld charges.

\subsection{Vibrational, dielectric, elastic and thermodynamic properties}

Thanks to the Density Functional Perturbation Theory, ABINIT can address directly the computation of linear and non-linear responses of the crystal to three types of perturbations: applied electric fields, applied strains, and 
internal atomic displacements. This is especially useful for the study of minerals, since the physical properties linked to such responses are numerous. Moreover, the implementation is valid for any type of symmetry, providing full tensors when needed. Let us review them.

Based on the linear responses (taking mixed perturbations into account), one can address the direct computation of the optical dielectric tensor, the static dielectric tensor, the elastic tensor (i.e. the 21 components in the triclinic case), the piezoelectric tensor, the Born effective (or dynamical) charges, the interatomic force constants, the dynamical matrices. From such data, the full vibrational spectrum (phonon band structure) can be computed. As a byproduct, one can obtain easily the infra-red spectrum (e.g. reflectivity), the frequencies of all the vibrational modes (Raman-active, infra-red active, or silent), the identification of LO and TO modes. More information on the capabilities opened by the availability of linear responses and the computational load of such calculations can be found in the review article on lattice dynamics of crystals, in the present issue [18].

Let us briefly exemplify such an application for a sorosilicate, the mineral åkermanite, $\mathrm{Ca}_{2} \mathrm{MgSi}_{2} \mathrm{O}_{7}$ [7]. Figure 1 shows the frequencies of computed zone-center vibrational modes and the measured Raman spectrum. There are many more theoretically predicted modes than the observed ones: Raman intensities might be small, or even vanish (due to symmetry reasons). The first-principles calculations allowed us to perform for each mode a detailed

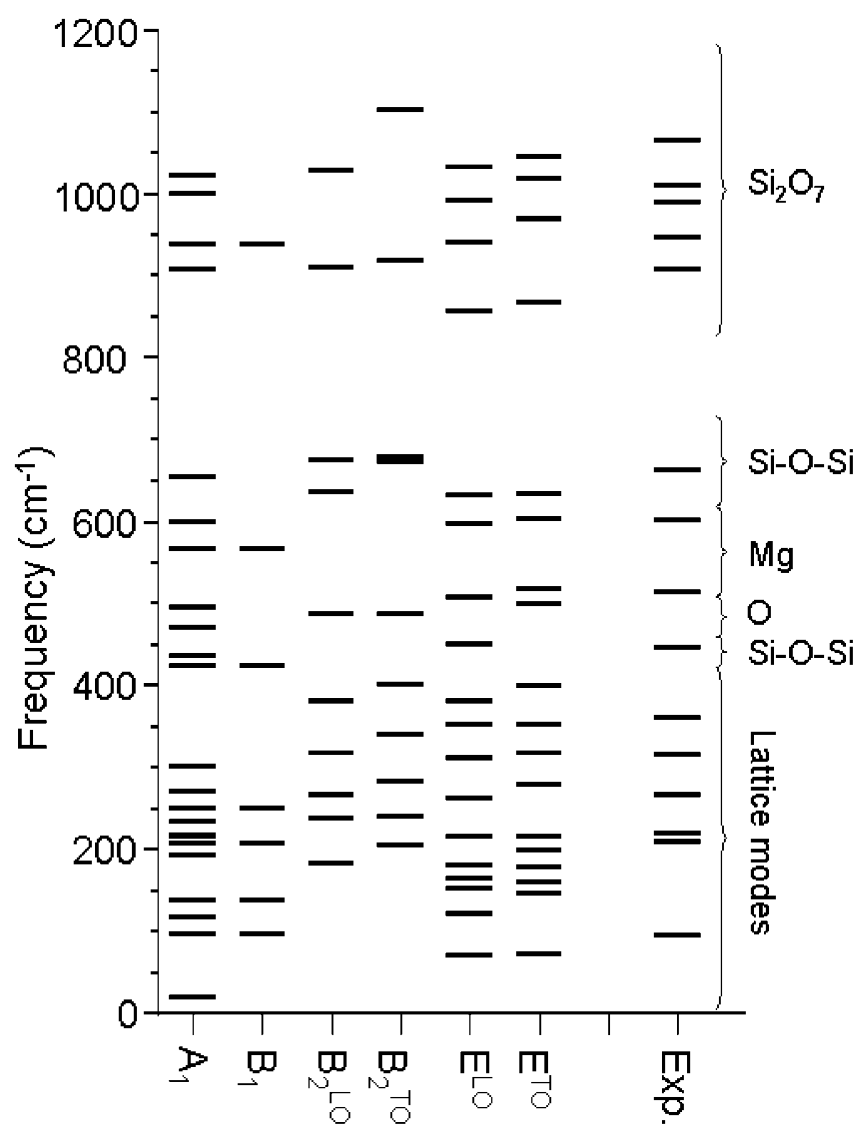

Fig. 1. Zone-center vibrational frequencies of åkermanite: theoretical frequencies, sorted by symmetry character $\left(\mathrm{A}_{1}\right.$ to $\left.\mathrm{E}^{\mathrm{TO}}\right)$, and experimental Raman lines. analysis of the associated displacement pattern, character and symmetry, and thus to interpret the experimental data. The modes below $400 \mathrm{~cm}^{-1}$ are lattice modes, the $448 \mathrm{~cm}^{-1}$ mode corresponds to the oscillation of the two $\mathrm{Si}$ atoms around $\mathrm{O}$ (with E symmetry character), the next modes up to $510 \mathrm{~cm}^{-1}$ are dominated by complex $\mathrm{O}$ displacements, the modes within the $510-635 \mathrm{~cm}^{-1}$ range correspond to $\mathrm{Mg}$ displacements. The mode characteristic of sorosilicates, observed around $664 \mathrm{~cm}^{-1}$ might be associated with the $655.9 \mathrm{~cm}^{-1}$ ( $\mathrm{A}_{1}$ symmetry) and $677.2 \mathrm{~cm}^{-1}\left(\mathrm{~B}_{2}\right.$ symmetry) modes. The higher frequency modes have complex displacement patterns involving mainly the $\mathrm{Si}_{2} \mathrm{O}_{7}$ group.

The phonon band structure proves to be a very effective tool for the study of stability of minerals. Indeed, if all the vibrational modes of a crystal are associated with a positive eigenvalue of the dynamical matrix, all the collective displacements of the atoms, or change of unit cells, increase the energy. Hence, the crystal is stable against all possible infinitesimal collective changes of its structure: an energy barrier separates this phase from other possible energetically favored phases. The full phonon band structure provides a unique and complete characterization of the (meta) stability of a phase. Examples of such phonon band structure computed with ABINIT can be found in the review article on incommensurate phases [21], and in the one presenting lattice dynamical properties of minerals [18], both in the present issue.

As a further step, the vibrational data can be used to characterize crystal thermodynamics at different temperatures, in the quasi-harmonic approximation: there is a direct link between the phonon band structure and thermodynamic data, such as entropy, specific heat and free energy [22]. Thus, the free energy of different phases can be compared, and P-T diagrams can be deduced. See for example Ref. [10], especially Fig. 2(b), or the above-mentioned review on lattice dynamical properties of minerals [18].

Finally, some non-linear responses are also available from ABINIT such as non-linear optical susceptibilities, electrooptic tensors and Raman efficiencies. The latter property is the most important one for mineralogy studies and is directly helpful to analyze the phonon spectrum.

\section{The user's point of view}

\subsection{Code availability}

ABINIT is available under the GNU General Public License (GPL), often referred to as a "Copyleft". Under this licensing scheme [23], the user receives four freedoms: (i) unlimited use of the package for any purpose; (ii) the freedom to study the sources and modify them to match his/ her needs; (iii) the freedom to copy the package; (iv) the freedom to distribute modified versions, if they are properly advertised as such. The license also protects these four freedoms by requiring that they must come with any transmitted copy of the program. Such a policy is especially valuable when the group of developers is large and there is a desire to let everybody benefit from and contribute to the project, which is the case with the ABINIT project. 
In practice, the source code of different versions of ABINIT (including the most recent ones) can be downloaded straightforwardly from the Web site [2], as well as the executables for many different platforms, as described now.

\subsection{Code portability}

Because the developers work on a variety of platforms (hardware/compiler), ABINIT has acquired a rather large portability. This is achieved through machine-dependent pre-processing, and a large set of automatic tests (more than 400 by now), that are used to check the correct execution on each platform. These tests can as well be used as a library of examples, to help the beginners to set up their input files.

Over the years, ABINIT has been installed on the following platforms: PC under Linux (with 4 different compilers), PC under Windows OS, Itanium 2, Opteron, Macintosh (OS X), Compaq/DEC alpha stations, HP, Sun, IBM, SGI Origin, NEC, Fujitsu, Hitachi, CRAY T3E. Corresponding executable codes are available on the Web site. The parallel version of ABINIT is available for most of these platforms as well (in particular, ABINIT is often used with PC clusters, but it has also been used on the largest computer in Europe, owned by the Commissariat à l'Energie Atomique).

\subsection{The tutorials}

Guided by the Web site, a newcomer will be able to learn how to use the basic functionalities of ABINIT without external help. At the heart of the beginner self-learning capabilities provided by ABINIT, one finds a set of six tutorials that have been designed precisely for this purpose, and have been found very effective worldwide: they are actually used in more than a dozen institutions, in solid-state physics lectures for undergraduate or graduate students. The tutorials cover all basic geometry optimization concepts, as well as the computation of response functions (at the most basic level) and the GW module of ABINIT.

\subsection{The documentation}

Beyond the tutorial, the documentation provided in the package is extensive. There are different manuals for the main program and the utilities, all available on the Web. The complete list of input variables is also provided on the Web, as well as the source of the program (with hyperlinks between different routines, allowing the sources to be browsed easily). Some complements to the theoretical formulations published in the literature are available in $\mathrm{T}_{\mathrm{EX}}$ format. Finally, the set of input files for the automatic tests, mentioned in Sec. 3.2, provides an outstanding source of documentation for the user: all the possible usages of ABINIT correspond to at least one example file.

\subsection{A few words about the future ...}

ABINIT being an open software project, relying on the good will of many different people, it is quite hard to make predictions about its evolution, beyond things that are pre- sently under development. As a follow-up of current development axes, more physical properties will be implemented. In particular, the computation of frequency-dependent linear and non-linear optical properties of crystals, as well as the prediction of properties based on electron-phonon coupling (like superconductivity, electrical resistivity, phonon lifetime ...) should be available in the next ABINIT release (ABINITv4.4). In a few year time, it seems reasonable to think that ABINIT will also be able to compute more thermodynamic properties, like the thermal expansion, or the thermal conductivity, Finally, one can bet that further improvements will concern the speed (better convergence algorithms, better parallelization), the robustness, the interchange of data with other codes (pseudopotential files, density files, wavefunction files ...).

Acknowledgments We thank all the contributors to the ABINIT project. In particular, without being exhaustive, let us mention computer scientists J.-P. Minet, L. Sindic, B. Van Renterghem, A. Roy, M. Boulet, B. Magne, as well as physicists or mineralogists S. Goedecker, F. Detraux, M. Fuchs, D. Vanderbilt, X. Wu, N. Hill, J.-C. Charlier, Na Sai, J. Junquera, P. Casek, F. Finocchi, Y.-M. Niquet, F. Tournus, J. Bouchet, K. Rabe, S. Erwin, M. Coté, J.-F. Brière, E. Matthis, A. Oganov, V. Recoules, G. Jomard, P. Thibaudeau, S. Bernard, G. Van Lier.

Several authors (X.G, G.-M. R., M.V., J.-Y. R.) thank the National Fund for Scientific Research (F.N.R.S.-Belgium) for financial support. This project has been supported by the Communauté Française de Belgique (through a subvention "Actions de Recherche Concertées"), by the Belgian Federal State ("Poles d'attraction Interuniversitaires", Phase V), by the European Union (contracts HPRNCT-2002-00317 ["EXCITING" Research Training Network "Firstprinciples approach to the calculation of optical properties of solids"], HPRN-CT-2000-00167 ["NANOPHASE" Research Training Network "Nanoscale photon absorption and spectroscopy with electrons"], and NMP4-CT-2004-500198 ["NANOQUANTA" Network of Excellence, "Nanoscale quantum simulations for nanostructures and advanced materials"]), and the French "Groupe de Recherche sur la Théorie de la Fonctionnelle de la Densité" (GDR-DFT).

\section{References}

[1] Gonze, X.; Beuken, J.-M.; Caracas, R.; Detraux, F.; Fuchs, M.; Rignanese, G.-M.; Sindic, L.; Verstraete, M.; Zerah, G.; Jollet, F.; Torrent, M.; Roy, A.; Mikami, M.; Ghosez, Ph.; Raty, J.-Y.; Allan, D. C.: First-principle computation of material properties: the ABINIT software project, Comput. Materials Science 25 (2002) 478-492.

[2] http://www.abinit.org

[3] Rignanese, G.-M.; Detraux, F.; Gonze, X.; Bongiorno, A.; Pasquarello, A.: Dielectric constants of $\mathrm{Zr}$ silicates: a first-principles study. Phys. Rev. Lett. 89 (2002) 117601.

[4] Oganov, A. R.; Gillan, M. J.; Price, G. D.: Ab initio lattice dynamics and structural stability of MgO. J. Chem. Phys. 118 (2003) 10174-10182.

[5] Clatterbuck, D. M.; Krenn, C. R.; Cohen, M. L.; Morris, J. W.: Phonon instabilities and the ideal strength of aluminum, Phys. Rev. Lett. 91 (2003) 135501.

[6] Verstraete, M.; Gonze, X.: First-principles calculation of the electronic, dielectric, and dynamical properties of $\mathrm{CaF}_{2}$. Phys. Rev. B68 (2003) 195123.

[7] Caracas, R.; Gonze, X.: Ab initio determination of the groundstate properties of $\mathrm{Ca}_{2} \mathrm{MgSi}_{2} \mathrm{O}_{7}$ akermanite. Phys. Rev. B68 (2003) 184102.

[8] Serrano, J.; Romero, A. H.; Manjon, F. J.; Lauck, R.; Cardona, M.; Rubio, A.: Pressure dependence of the lattice dynamics of $\mathrm{ZnO}$ : An ab initio approach. Phys. Rev. B69 (2004) 094306.

[9] Finocchi, F.; Barbier, A.; Jupille, J.; Noguera, C.: Stability of rocksalt (111) polar surfaces: Beyond the octopole. Phys. Rev. Lett. 92 (2004) 136101. 
[10] Oganov, A. R.; Ono, S.: Theoretical and experimental evidence for a post-perovskite phase of $\mathrm{MgSiO}_{3}$ in earth's D" layer. Nature 430 (2004) 445-448.

[11] Hohenberg, P.; Kohn, W.: Inhomogeneous electron gas. Phys. Rev. 136 (1964) B864-B871.

[12] Kohn, W.; Sham, L. J.: Self-consistent equations including exchange and correlation effects. Phys. Rev. 140 (1965) A1133A1138.

[13] Baroni, S.; Giannozzi, P.; Testa, A.: Green's-function approach to linear response in solids. Phys. Rev. Lett. 58 (1987) 18611864.

[14] Gonze, X.; Allan, D. C.; Teter, M. P.: Dielectric tensor, effective charges and phonon in $\alpha$-quartz by variational density-functional perturbation theory. Phys. Rev. Lett. 68 (1992) 3603-3606.

[15] Gonze, X.: First-principle responses of solids to atomic displacements and homogeneous electric fields: implementation of a conjugate-gradient algorithm. Phys. Rev. B55 (1997) 1033710354.

[16] Gonze, X.; Lee C.: Dynamical matrices, Born effective charges, dielectric permittivity tensors, and interatomic force constants from density-functional perturbation theory. Phys. Rev. B55 (1997) 10355-10368.

[17] Baroni, S.; de Gironcoli, S.; Dal Corso, A.; Giannozzi, P.: Phonons and related crystal properties from density-functional perturbation theory. Rev. Mod. Phys. 73 (2001) 515-562.

[18] Gonze, X.; Rignanese, G.-M.; Caracas, R.: First-principle studies of the lattice dynamics of crystals, and related properties. $\mathrm{Z}$. Kristallogr. 220 (2005) 458-472.

[19] Aulbur, W. G.; Jonsson, L.; Wilkins, J. W.: Quasiparticle calculations in solids. Solid State Physics 54 (2000) 1-218.

[20] Blöchl, P. E.: Projector augmented-wave method. Phys. Rev. B50 (1994) 17953-17979.

[21] Caracas, R.; Gonze, X.: First-principle study of materials involved in incommensurate transitions. Z. Kristallogr. 220 (2005) $511-520$.

[22] Lee, C.; Gonze, X.: Ab initio calculation of the thermodynamical properties and atomic temperature factors of $\mathrm{SiO}_{2} \alpha$-quartz and stishovite. Phys. Rev. B51 (1995) 8610-8613.

[23] http://www.gnu.org/copyleft/gpl.txt 